\title{
1 Active coacervate droplets are protocells that grow and resist Ostwald ripening
}

3 Karina K. Nakashima ${ }^{1}$, Merlijn H. I. van Haren ${ }^{1}$, Alain A. M. André ${ }^{1}$, Irina Robu ${ }^{1}$ and 4 Evan Spruijt $t^{*}$

61 Institute for Molecules and Materials, Radboud University, Heyendaalseweg 135, 76525 AJ Nijmegen, the Netherlands.

$9{ }^{*}$ Correspondence: e.spruijt@science.ru.nl

11 Keywords: protocells, complex coacervates, artificial growth model, active droplets

\section{Abstract}

14 Active coacervate droplets are liquid condensates coupled to a chemical reaction that turns over

15 their components, keeping the droplets out of equilibrium. This turnover can be used to drive active

16 processes such as growth, and provide an insight into the chemical requirements underlying

17 (proto)cellular behaviour. Moreover, controlled growth is a key requirement to achieve population

18 fitness and survival. Here we present a minimal, nucleotide-based coacervate model for active

19 droplets, and report three key findings that make these droplets into evolvable protocells. First, we

20 show that coacervate droplets form and grow by the fuel-driven synthesis of new coacervate

21 material. Second, we find that these droplets do not undergo Ostwald ripening, which we attribute

22 to the attractive electrostatic interactions within complex coacervates, active or passive. Finally,

23 we show that the droplet growth rate reflects experimental conditions such as substrate, enzyme

24 and protein concentration, and that a different droplet composition (addition of RNA) leads to

25 altered growth rates and droplet fitness. These findings together make active coacervate droplets a

26 powerful platform to mimic cellular growth at a single-droplet level, and to study fitness at a 27 population level. 
29 Growth and division are essential processes in life, without which we cannot explain survival and 30 reproduction. Modern cells rely on tightly coordinated mechanisms involving complex machinery,

31 but even primitive cells without specialized enzymes and proteins already succeeded in 32 proliferating. This suggests that the behaviour can be reproduced (and explained) using solely 33 chemical principles. ${ }^{1,2}$ Such principles may shed light on the emergence of the first cells and. 34 Moreover, they help broadening the scope of chemical models used to mimic and decipher 35 biological behaviour. ${ }^{3}$ One of the simplest systems predicted to exhibit growth and division is a 36 droplet coupled to a chemical reaction: by keeping the reaction out of equilibrium (e.g., with a 37 supplied fuel), the droplet can sustain an active behaviour like growth (i.e., an active droplet). ${ }^{4-9}$ 38 To ensure that the reaction can directly influence behaviour, the droplet must be an open compartment able to exchange material with its surroundings, and compatible with volume change. Coacervates are a promising system to fulfil these requirements. ${ }^{10,11}$

Coacervate droplets form spontaneously by phase separation in a saturated solution of

42 macromolecules; when the phase separation is driven by attractive electrostatic interactions, they

43 are called complex coacervates. Coacervates lack a membrane and thus have no physical barrier

44 that limits their growth. The droplets are permeable to molecules from the surroundings with some 45 selectivity, and concentrate the solutes through dynamic interactions, opening the way for its 46 building blocks to be synthesized in situ. ${ }^{12}$ As coacervate droplets are governed by liquid-liquid 47 phase separation, they are tied to equilibrium concentrations of the building blocks, and the volume 48 of one phase can grow while the internal concentration remains constant, which aligns perfectly 49 with the active droplet requirements. This is crucial as most protocell models so far have increased 50 in size via passive mechanisms: vesicle fusion, ${ }^{13}$ droplet coalescence and ripening, ${ }^{14,15}$ or uptake 51 of externally added building blocks. ${ }^{16}$

Coacervates can achieve growth more easily than vesicles, but are still subject to passive processes. Brownian-motion-induced coalescence and Ostwald ripening can compete with, or 
mask, reaction-diffusion limited growth, ${ }^{17}$ and although these processes also lead to an increase in average droplet volume, this growth comes at the expense of a decreased droplet number completely disconnected from biological growth. Therefore, for coacervates to hold any potential as dynamic biomimetic models, it is crucial to develop a stable, active system. In addition, growing coacervates must be studied quantitatively and at a single-droplet level in order to undoubtedly distinguish active growth from passive coarsening. We thus set out to develop an active coacervate model, i.e. one that grows like cells do in two senses: via an increase in droplet volume while keeping droplet count constant (growth), or via an increase in droplet count (nucleation). ${ }^{18}$ Our experimental model for active droplets is based on the pyruvate kinase-catalyzed conversion of ADP into ATP that we published previously (Figure 1). ${ }^{19}$ The efficiency of the enzymatic reaction allows us to avoid side reactions (keeping the system simple) and control the reaction rate - fast enough to overcome passive coarsening, and slow enough to avoid spinodal decomposition. ${ }^{20}$ Additionally, partitioning of the kinase offers an insight into the location of the

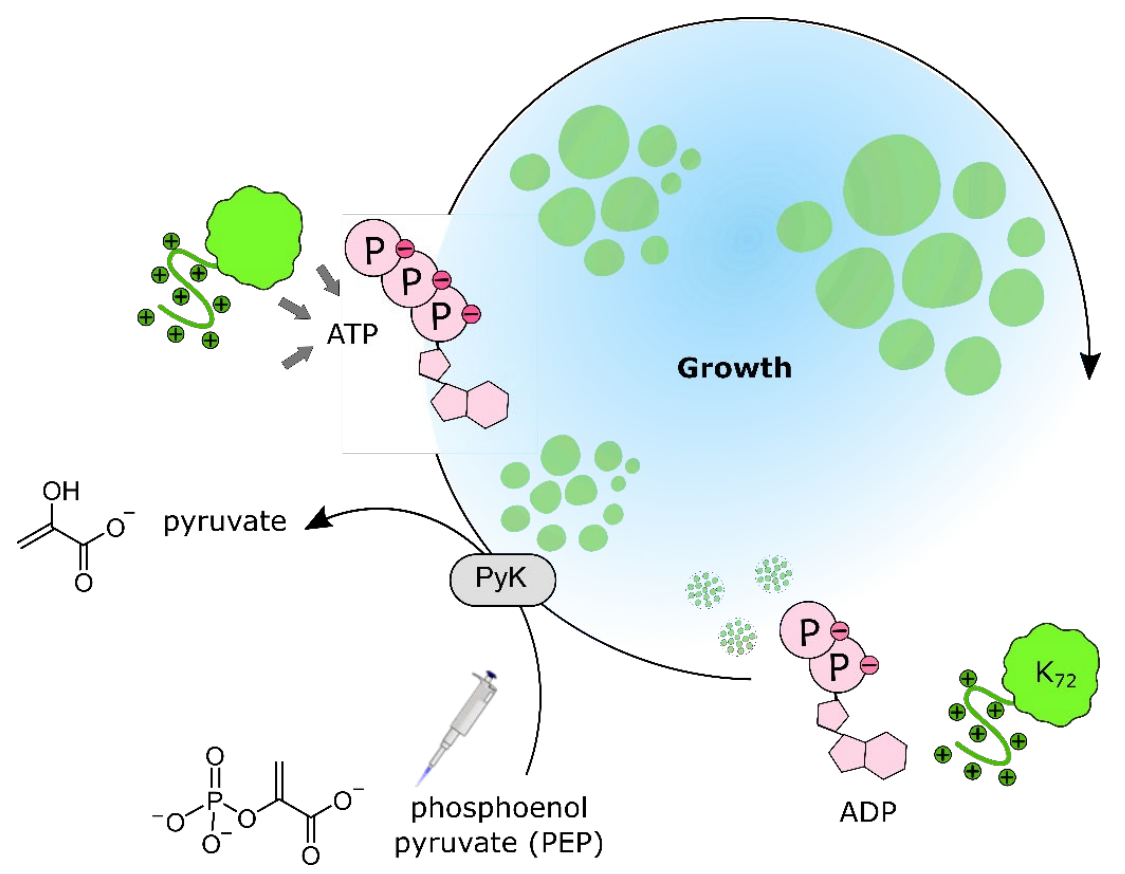

Figure 1. Active droplets scheme. The pyruvate kinase-catalyzed (PyK) conversion of ADP to ATP, combined with the liquid-liquid phase separation of ATP- $\mathrm{K}_{72}$ complexes, is a minimal translation of an active droplet. In this system, ADP is the substrate, and ATP (together with the lysine-rich protein $\mathrm{K}_{72}$ ) is the droplet material. We fuel the droplets by a manual addition of the second substrate, PEP. The waste, pyruvate, is not re-used in our setup. The local increase in the amount of ATP inside the droplets causes recruitment of more protein, leading to droplet growth. Growth may compete with other active (nucleation) and passive processes (coalescence, Ostwald ripening) that need to be distinguished experimentally. 


4

reaction. We analyse the growth at a single-droplet level, opening the way to investigate the dynamics of individual membrane-less protocells. We show that droplets grow actively driven by the enzymatic reaction, leading to a significant increase in size. In some conditions, nucleation is preferred over growth and droplet count increases. The droplets exhibit a common growth profile that can be rationalized in terms of protein diffusion, triggered by the reaction. By isolating the contributions of active and passive processes to droplet size evolution, we find that our complex coacervate droplets do not undergo Ostwald ripening and can remain stable for observation for more than an hour. Finally, under the same environmental conditions, droplets of different compositions grow at different rates, opening the way for the design of evolvable protocells.

\section{Results}

ATP-based coacervates have previously been studied as dynamic membrane-less protocells compatible with growth, enzymatic reactions and RNA partitioning. ${ }^{21,22}$ Inspired by the phosphorylation-mediated liquid-liquid phase separation of peptide-RNA developed by the group of Keating, ${ }^{23}$ our group achieved reversible ATP-poly- $L$-lysine coacervates with the introduction of pyruvate kinase (PyK) to generate ATP in situ from ADP and phosphoenolpyruvate (PEP). ${ }^{19}$ With the high efficiency of the PyK reaction and lack of side reactions that can overcomplicate non-enzymatic systems, we hypothesized we could achieve enough control of coacervation to obtain a coordinated behaviour like growth (see scheme in Figure 1). In comparison to our previous work, we replaced poly- $L$-lysine by $\mathrm{K}_{72}$ as a cationic fluorescent protein, which has already been used to form droplets with RNA. ${ }^{24} \mathrm{~K}_{72}$ contains 72 repeats of the pentapeptide VPGKG (an elastinlike sequence $)^{25,26}$ and is labelled with green fluorescent protein (GFP). It can form condensates at low concentrations with ATP, which can be easily monitored by fluorescence microscopy. 
The first step in the design of our system was to determine the conditions under which ATP

100 (the droplet material), but not ADP (the substrate), forms droplets with the $\mathrm{K}_{72}$ protein (Figure $2 \mathrm{~A}$ ).

101 This "coacervation window" is the range of conditions where ATP-K $\mathrm{K}_{72}$ droplets can nucleate and

102 grow as a result of conversion of ADP into ATP. By measuring the phase diagram in terms of salt

103 concentration (Figure 2B), we estimate the stability of coacervate droplets to a chemical reaction

104 that produces charged by-products - in this case, the pyruvate kinase-catalyzed formation of ATP

105 also generates pyruvate. The typical phase diagram of ADP/ATP-K $\mathrm{K}_{72}$ complex coacervates shows 106 that at $3 \mathrm{mM}$ of nucleotide and no added salt, the difference between ADP and ATP in affinity for $107 \mathrm{~K}_{72}$ is maximal, which is ideal to translate progress of the chemical reaction into a volume change. We further determined the partitioning coefficient $\left(K_{\mathrm{p}}\right)$ of the main reaction species to create

109 a kinetic map of our system (Figure 2C). We prepared ATP-K 72 droplets as hosts and added labelled 110 pyruvate kinase and ADP as client molecules. As expected, the $K_{\mathrm{p}}$ of ATP (2.8) is higher than that 111 of ADP (1.1), but even above the critical salt concentration of ADP-K $\mathrm{K}_{72}$, ADP can be incorporated 112 as a client (Supplementary figure 1). To determine enzyme $K_{\mathrm{p}}$, we labelled it with Alexa Fluor-647 113 maleimide, targeting exposed cysteines. We chose a cysteine-reactive label to avoid modification 114 of charged residues (lysines), which can affect the partitioning (Supplementary figures 2-3). Based 115 on the measured partitioning coefficients (Figure 2C), and the fast fluorescence recovery 116 (Supplementary figure 4), we can make the following assumptions: i) ADP can enter the droplets 117 if they become depleted of it; ii) ATP, PyK and $\mathrm{K}_{72}$ accumulate inside the droplets and can exchange 118 with the surroundings; and iii) the reaction can occur inside the droplets, where the enzyme is 119 concentrated. These are key requirements to keep the system out of equilibrium with a supply of 120 substrate and attain reaction-driven growth. 
A
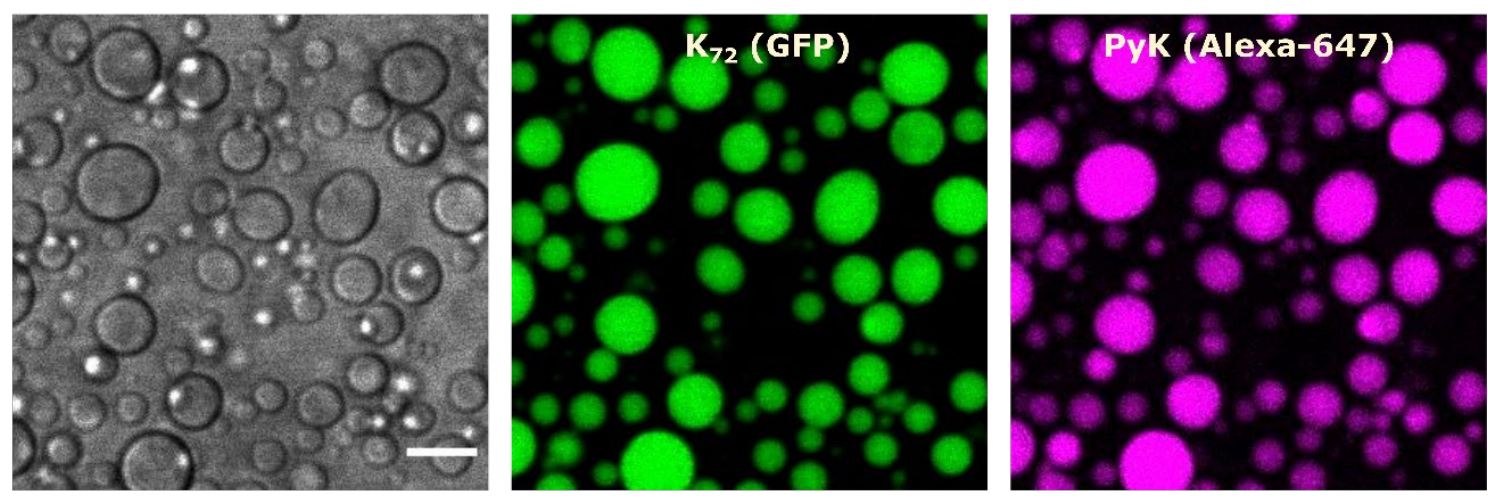

B

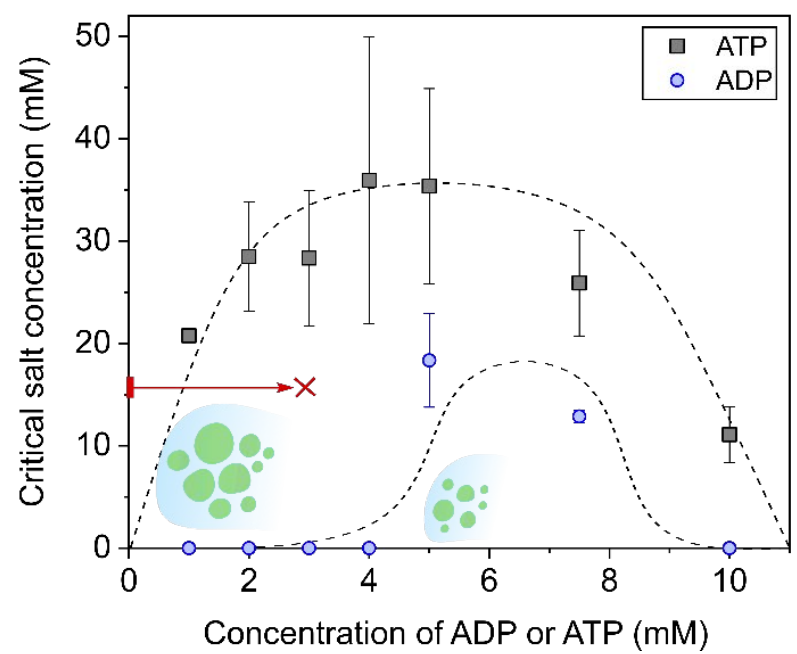

C

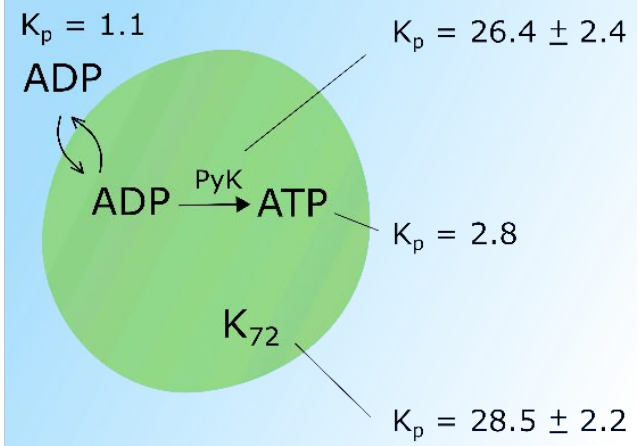

Figure 2. Main properties of ATP- $\mathrm{K}_{72}$ coacervate droplets. (A) ATP-K $\mathrm{K}_{72}$ droplets containing Alexa Fluor-647 labelled pyruvate kinase. Channels are shown separately: gray (left) - transmission, green (middle) - GFP (attached to $\mathrm{K}_{72}$ ), magenta (right) - Alexa Fluor-647. $\mathrm{K}_{72}$ always contains the GFP tag; PyK was labelled with Alexa-647 only for this experiment. Scale bar: $10 \mu \mathrm{m}$. (B) The phase diagrams of ADP- $\mathrm{K}_{72}$ and ATP-K $\mathrm{K}_{72}$ mixtures confirm that the conversion of ADP to ATP can induce coacervation under certain conditions and lead to growth (e.g. along the red line). The dashed lines representing the approximate phase boundaries are meant as a guide to the eye. (C) The partitioning coefficients of the main components (measured via HPLC or fluorescence) are in accordance with Figure 1.

\section{Single-droplet analysis of coacervates over time}

After mapping out the conditions under which active droplets could exist, we investigated

if a fuel-driven reaction could bring about active growth as a step towards evolvable protocells.

Taking advantage of the fluorescence from the $\mathrm{K}_{72}$ proteins condensed inside the coacervates, we

134 can monitor the evolution of individual coacervates nucleating, growing and resting on a plane

135 above the glass surface for at least an hour with confocal laser scanning microscopy

136 (Supplementary figure 5). To gain a fitness advantage, actively growing protocells must be able to overcome passive coarsening, occurring through coalescence or Ostwald ripening. We first compared passive pre-formed ATP-K 72 droplets at high and low volume fraction, in which we 
expected coalescence and Ostwald ripening at varying intensity, with active droplets growing by

140 conversion of ADP into ATP. In our setup, by directly tracking droplet size, fusion events are not

141 mistaken for growth, but it remains important to establish the conditions under which active growth

142 can outcompete passive coarsening. We detect the droplets by their boundaries and extract

143 properties such as area, centroid position, circularity and total fluorescence intensity. We label

144 droplets by their centroid and then build a profile of radius over time, where each droplet has its 145 own curve (Supplementary figure 6).

146 In a high-volume-fraction passive system (3 $\mathrm{mM} \mathrm{ATP,} 20 \mu \mathrm{M} \mathrm{K} \mathrm{K}_{72}$, estimated volume 147 fraction ca. 1\%), most droplets exhibit steps in the radius profile (Figure 3A). At this volume 148 fraction, frequent coalescence events lead to (discrete) increases in droplet volume of tens of fL $149\left(\mu \mathrm{m}^{3}\right)$ every hour, ${ }^{17}$ although the droplet count does not decrease due to simultaneous gravitational 150 settling from the top of the solution to the glass plane. The volume fraction, and hence coalescence, 151 can be controlled by adjusting the concentration of the components.

At a lower droplet density ( $1 \mathrm{mM}$ ATP, $20 \mu \mathrm{M} \mathrm{K}_{72}$, estimated volume fraction $\left.0.3 \%\right)$, most 153 passive droplets show a stable size (Figure 3B) that can persist for an hour (Supplementary figure 154 7). We observed significantly fewer coalescence events, as expected, but surprisingly, we also 155 observed no measurable Ostwald ripening in the form of gradual expansion of large droplets and 156 shrinkage of small droplets, even though we expected clear ripening according to our most 157 conservative estimates of the ripening parameters (Supplementary table 2). The absence of Ostwald 158 ripening, which we explain in more detail in the following section, is a remarkable behaviour and 159 of great importance for our goal to achieve active growth in very small coacervate droplets. Based on our findings with passive droplets, we were hopeful to observe distinctly different 161 kinetic traces for active droplets at low volume fractions. For ATP-K 72 droplets forming by chemical 162 conversion from ADP, the initial volume fraction is even smaller than that in Figure 3B. Coalescence 163 will therefore be even less frequent and is not expected to mask the onset of active growth. Indeed, 164 the profiles of active growth (Figure 3C) are clearly distinct from the two sets of passive profiles 

with PEP, droplets of $0.5 \mu \mathrm{m}$ radius started forming within a minute. Especially at the initial times, the vast majority exhibited a continuous growth curve (Figure 3C). Importantly, in contrast to passive droplets coarsening, growth does not compromise persistence and the droplet count in this case can increase (as shown in Figure 3C and Supplementary figure 9).
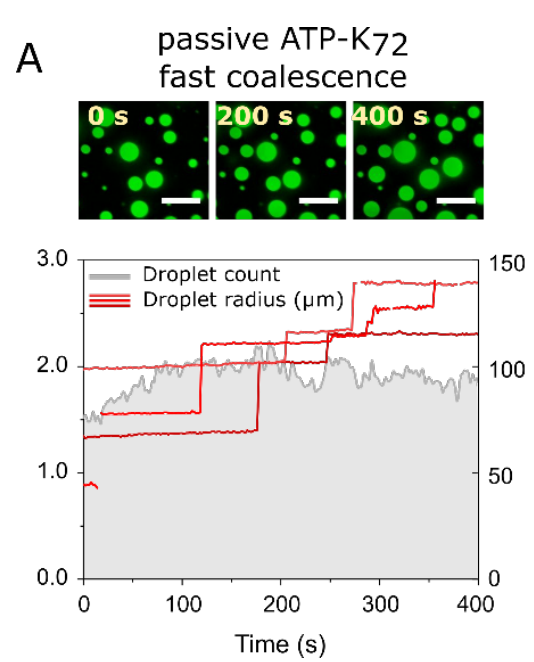
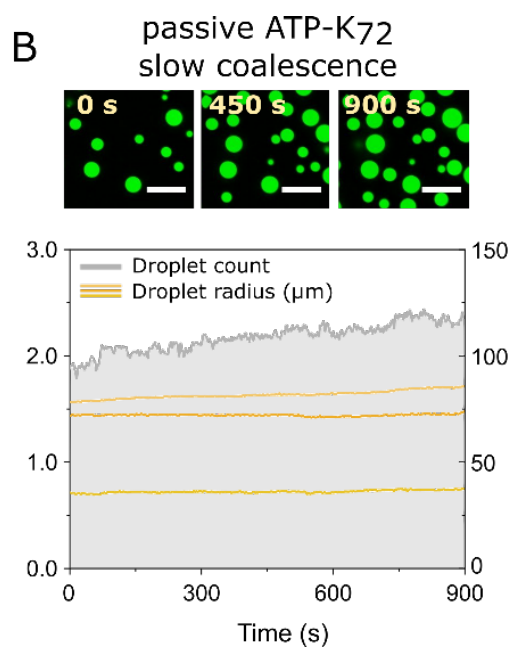
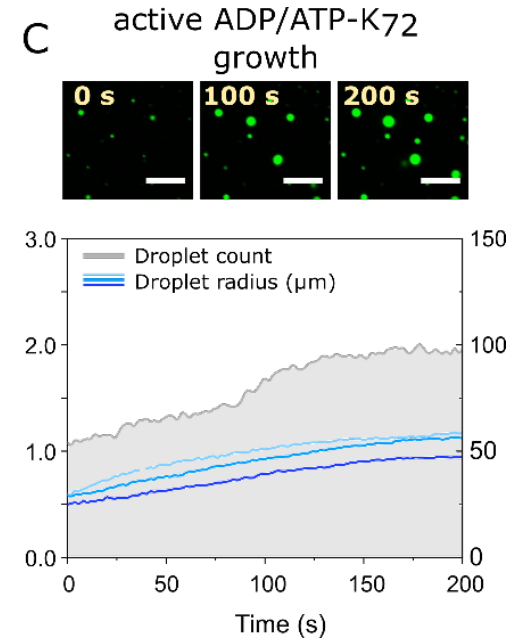

Figure 3. Passive and active droplets in radius profiles. (A) Passive coacervate droplets exhibit discrete increases in radius or (B) at lower volume fraction can remain stable for minutes. (C) The gradual increase in droplet radius over time is characteristic of active droplets, for which also the droplet count increases. All: left axes indicate droplet radius (in $\mu \mathrm{m}$ ) and right axes indicate droplet count. Scale bars are $10 \mu \mathrm{m}$. For visual clarity, only three exemplary traces were chosen out of each experiment. Full frames can be found in Supplementary figure 8.

\section{Suppressed Ostwald ripening of complex coacervate droplets}

We noticed that - surprisingly - the active and passive droplets did not show any ripening, despite prolonged observation. Instead, droplets were found to remain stable for at least an hour. Ostwald ripening has been predicted to be suppressed in active emulsions, where a chemical reaction (with the appropriate rate) causes a bigger efflux of molecules from large droplets than from small ones. ${ }^{27}$ Experimentally, however, active oily droplets coupled to an anhydride formation reaction exhibit accelerated ripening, as the reaction activation/deactivation flux adds to the diffusive flux between droplets. ${ }^{9}$ In our experiments with either passive or active coacervate droplets, we did not observe any shrinkage of small droplets, suggesting that Ostwald ripening is being slowed down or prevented by an opposing force closely linked to the nature of our droplets. ${ }^{28}$ 
To understand why these complex coacervate droplets would not show ripening, we

188 consider the balance of (thermodynamic) forces underlying Ostwald ripening. The components of

189 a complex coacervate droplet have a finite, usually low solubility in the continuous dilute phase.

190 In our case, the solubility of $\mathrm{K}_{72}$ (molecular volume of ca. $\left.65 \mathrm{~nm}^{3}\right)^{29}$ is the lowest at about $5 \mu \mathrm{M}$,

191 which creates a kinetic barrier for ripening. Based on these broad estimates, we expect that these

192 coacervate droplets could ripen 3-7 $\mu \mathrm{m}$ in radius every hour. ${ }^{17}$ The droplets tracked have a radius

193 of 0.4 to $3 \mu \mathrm{m}$, but analysis of size over time, local rates, size-rate correlation and droplet count do

194 not agree with a ripening profile, suggesting that ripening is not only slow, but suppressed. We

195 verified that this was not a limitation of our experimental setup by performing positive controls

196 with passive oil droplets of 1-bromo-dodecane and 1-bromo-propane, for which we were able to

197 visualize shrinkage (depicted as a negative growth rate in our analysis) and decaying droplet count

198 (Figure 4B).

Therefore, we hypothesize that complex coacervates are special liquids that exhibit 200 suppressed Ostwald ripening, because they are formed via associative phase separation. Typical 201 ripening is driven by the increased Laplace pressure inside small droplets $\left(U_{\mathrm{c}}\right.$ in Figure 4$)$, but 202 ignores the required disruption of attractive interactions when charged molecules diffuse out of the 203 droplet. The separation of a positively charged $\mathrm{K}_{72}(Q=+65 e)$ from a coacervate droplet of size $R$ 204 will leave a residual negative surface charge density of $-Q / 4 \pi R^{2}$, which comes with an electrostatic 205 penalty that is larger for smaller droplets $\left(U_{\mathrm{e}}\right.$ in Figure $\left.4 \mathrm{C}\right)$. Weighing that penalty against the 206 Laplace pressure difference that drives Ostwald ripening, we find that the exchange of material 207 between complex coacervate droplets may not necessarily occur in the direction from small to large 208 droplets. With typical estimates of the surface tension, molecular volume and Debye length in our 209 ATP-K 72 coacervate droplets, the transfer of charged material from one droplet to another is 210 endergonic regardless of the relative radii (Figure 4D and Supplementary table 3).

211 This suggests that Ostwald ripening can be suppressed by the nature of the interactions 212 underlying droplet formation, and that many complex coacervates can persist for extensive times, 
provided that the charge of the building blocks is large enough. Indeed, our experiments indicate

214 that Ostwald ripening is absent in both passive and active complex coacervate droplets, and we

215 confirmed that Ostwald ripening was also absent in another, passive complex coacervate system

216 (Supplementary figure 10). Importantly, from a protocell perspective this means that if we

217 introduce an active process in these slow-ripening, slow-fusing droplets, the resulting active

218 droplets could mimic cellular growth without interference from passive coarsening processes, and

219 the growth can be controlled by the same parameters that control a chemical reaction.

A

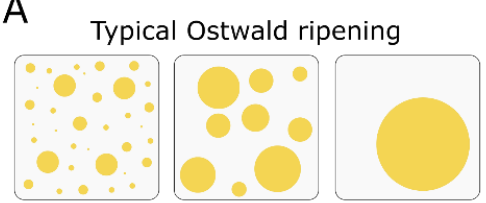

Complex coacervate droplets

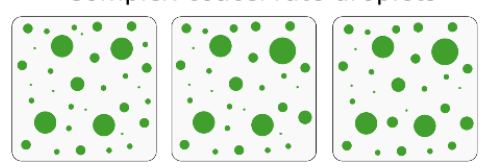

B

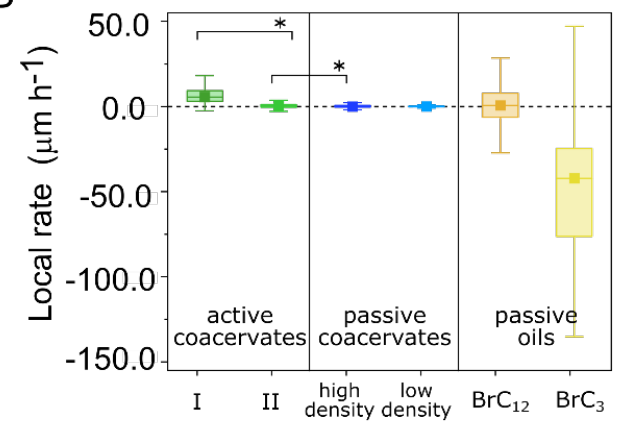

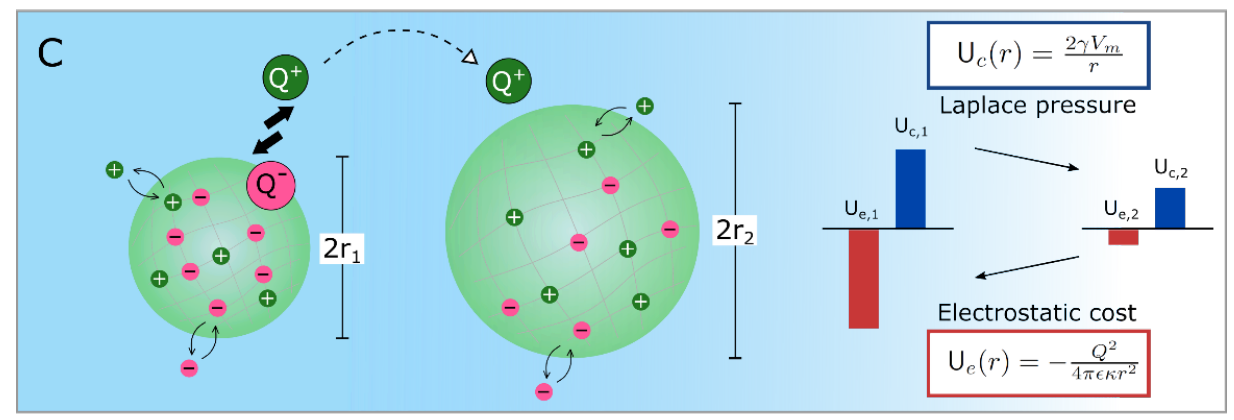
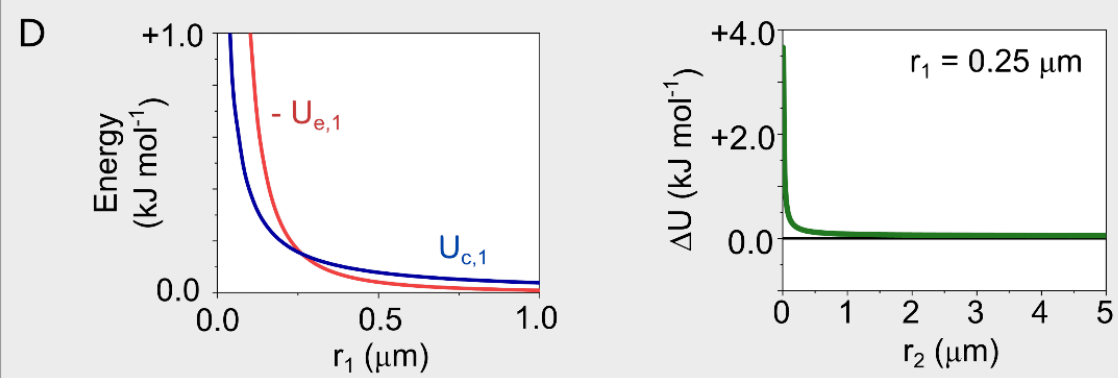

Figure 4. Ostwald ripening in complex coacervates. (A) Schematics of the distinct behaviour observed for oil-based droplets and complex coacervate droplets. (B) The local rates can be used to quantify that distinction: active coacervates grow (phase I and II are discussed below in Figure 5), and passive coacervates (high or low droplet densities) remain stable in size; droplets of 1-bromopropane and 1-bromododecane shrink, or exhibit a negative local rate. (C) Rationalization of suppressed Ostwald ripening in complex coacervates, taking into account both Laplace pressure $\left(U_{c}\right)$ dependence on radius and the electrostatic barrier $\left(U_{e}\right)$ to removing a soluble (highly) charged molecule from a coacervate droplet. (D) Our proposed model for suppressed ripening shows that the potential energy of a charged molecule near a complex coacervate droplet has comparable contributions of the electrostatic and chemical components, and that the sign of the transfer energy of the molecule to neighbouring droplets $(\Delta U)$ may be independent of the relative radii. 
Having established that ATP-K 72 complex coacervate droplets show negligible Ostwald

233 ripening on the timescale of our interest, we return to the active droplets of Figure $3 \mathrm{C}$ to obtain a

234 better understanding of the active growth. We find that the droplets start growing only after the fast growth, seemingly of a linear increase of radius with time; around 5 minutes growth slows down, and after 10 minutes most droplets have reached a plateau of stable size, as can be seen in Figure 5A 239 (extended curves in Supplementary figure 11). The plateau coincides with the depletion of fuel, as indeed, if fuel is re-supplied, the droplets can regain growth (Figure 5B).

A

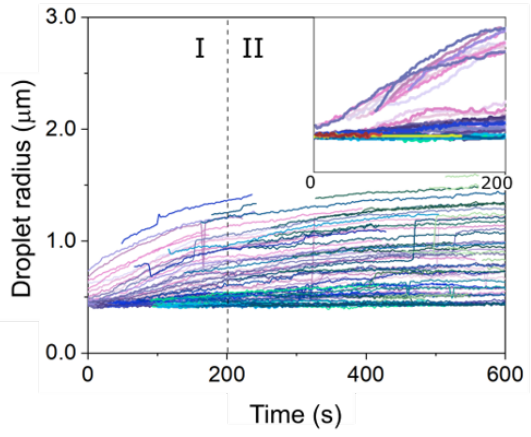

B

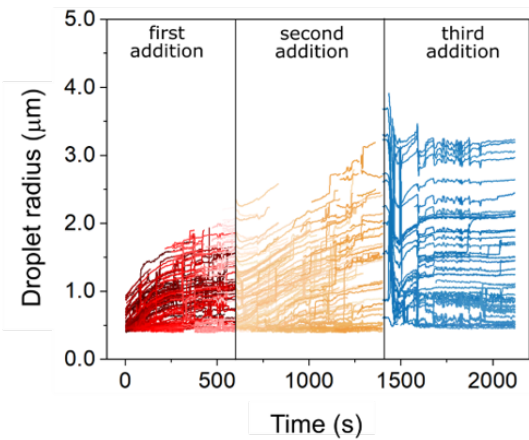

C

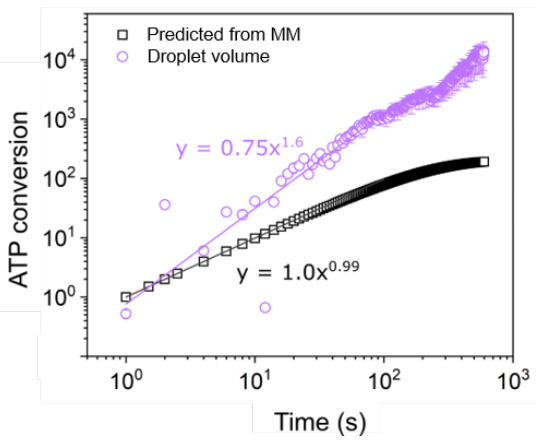

Figure 5. Growth of active droplets. (A) Radius traces of all droplets in a selected active droplet experiment (original: video 6). In the inset, the curves were shifted horizontally for better visualization of common behavior. The color scheme red-to-yellow reflects droplets that were detected earlier-to-later on the experiment. (B) Stepwise addition of fuel (PEP) to active droplets. In each step, $1 \mathrm{mM}$ of PEP was added, after the growth curve plateau was reached. Original videos: 9, 10 and 11 respectively. (C) Profile of the ATP conversion based on average droplet volume evolution (calculated from dataset in A), compared to the profile estimated based on Michaelis-Menten kinetics in solution, using $\mathrm{k}_{2}$ of $0.3 \mathrm{~min}^{-1}$ and ADP starting concentration of $3 \mathrm{mM}$. The solid lines are power-law fits to the initial 50 seconds of growth. The calculated conversions have been normalized such that the initial slopes cross at $(1,1)$. Note that the ATP conversion in growing droplets and solution cannot be compared directly, since the exact droplet volume fraction is not known.

At a first glance, each droplet seems to have a unique trace, but that is mainly caused by the polydispersity in droplet size. All curves have the same overall shape and if horizontally shifted, a universal growth profile becomes evident (inset in Figure 5A), which is an indication that a 
show a separate group of traces and are always delayed (i.e., they start to grow when their size exceeds the $0.5 \mu \mathrm{m}$ threshold radius). This delay becomes more evident at lower enzyme

260 concentrations (Supplementary figure 13), suggesting that these small droplets might lack any 261 enzyme at all and rely solely on the incorporation of ATP produced in the dilute solution. Indeed,

262 if we estimate the inner enzyme concentration based on a total of $0.42 \mu \mathrm{M}$, a $K_{\mathrm{p}}$ of ca. 20 and a $1 \%$ 263 volume fraction of droplets, the average number of enzymes in a $0.5 \mu \mathrm{m}$ radius droplet is 2 . Once 264 these droplets surpass a threshold size at which they contain a higher enzyme count, they could 265 start to grow more rapidly and their radius increases close to linearly in time.

To explain the observed growth profile, we consider the kinetics involved in droplet

267 nucleation and growth. Once the first droplets are formed by nucleation (or if we add a small 268 amount of pre-existing ATP-K ${ }_{72}$ droplets), the reaction can happen in two phases: droplet and 269 surroundings. For droplets that nucleate at a threshold size beyond $0.5 \mu \mathrm{m}$, the reaction taking place 270 inside the droplets is dominant. Although we are not able to measure the effective in-droplet $k_{\text {cat }}$ of 271 PyK, we reason it is at least the same as for free enzyme, based on HPLC measurements of PyK 272 kinetics in the presence of coacervates (Supplementary figure 12), ${ }^{30}$ in which case the high inner 273 ADP and PyK concentrations would be sufficient for a faster reaction in the droplets. This 274 behaviour is still fundamentally different from the classic enzyme kinetics of PyK in solution: in 275 those cases, the amount of ATP produced is initially linear and decreases as substrates are being 276 depleted and ATP reversibly inhibits the enzyme. ${ }^{31}$ Inside complex coacervate droplets, inhibition 277 by ATP has a much smaller effect on enzyme activity, possibly because it remains bound to the 278 positively charged $\mathrm{K}_{72}$.

The conversion of ADP into ATP inside the droplets results in a continuous replenishment 280 of ADP and uptake of additional $\mathrm{K}_{72}$ and PyK to maintain partitioning equilibrium. If transport of 281 those compounds would be fast compared to the reaction, we expect that the amount of new ATP 282 produced is directly proportional to the actual volume of the coacervate droplet, leading to an 283 exponential increase in droplet volume (and radius) in time, analogous to the kinetics of a pure 
autocatalytic reaction. ${ }^{32}$ However, in our case the droplet size does not increase exponentially in time, suggesting that transport of building blocks from the surroundings into the droplet is limiting the growth.

Of all building blocks, $\mathrm{K}_{72}$ and $\mathrm{PyK}$ are the largest compounds, present at relatively low concentrations compared to ADP, and the slowest to diffuse. As $\mathrm{K}_{72}$ is required as droplet material to compensate the excess charges of ATP produced inside the droplets, we reason that transport of $\mathrm{K}_{72}$ limits the growth of droplets. The flux of molecules across the interface is proportional to the surface area $\left(4 \pi R^{2}\right)$ and the concentration gradient at the interface $\left(\mathrm{d}\left[\mathrm{K}_{72}\right] / \mathrm{d} R\right)$. This situation is analogous to the growth of condensed cloud droplets in a saturated vapour phase, and the radial growth is predicted to follow: $R(t)=\left(R_{0}+2 \xi t\right)^{1 / 2}$ after nucleation, where $\xi$ is a function of the supersaturation of the environment, which is set in our case by the concentration of $\mathrm{K}_{72}$ in solution and the reaction rate. ${ }^{33}$ For simplicity, we assume that $\xi$ is constant in a short interval of time, and we find that the droplet volume will increase as $V(t)=(4 \pi / 3)\left(R_{0}+2 \xi t\right)^{3 / 2}$, in perfect agreement with our results in Figure 5C. In short, the active droplets in our experiments grow as a result of an autocatalytic conversion of ADP into ATP, but the overall growth is limited by the diffusion of $\mathrm{K}_{72}$ from the surrounding solution to the droplet interface, where it can be taken up. We note that transport of other compounds, including PyK and PEP, could also limit the growth when their concentrations are altered. However, this would only change the growth rate constant $\xi$ and not change the scaling of droplet size in time, as these compounds must also be transported by diffusion to the droplet interface. ${ }^{5,27}$

\section{Growth at a population level}

In order to corroborate our model and analyse the effects of varying the concentrations of fuel, catalyst and building blocks, we need to quantify the typical growth rate (the "fitness") of an entire population of droplets. Since the droplets vary in size but show a universal growth profile (Figure 5A), we chose to average their local growth rates, defined as the variation in radius across 
310 a time interval, given in units of $\mu \mathrm{m} \mathrm{h}^{-1}$. The local rate is measured over small intervals of $20 \mathrm{~s}$,

311 during the first 2 minutes of the reaction. We analyse hundreds of droplets together in every

312 experiment and found that also at the population level active droplets have a distinct behaviour

313 from passive droplets. The distance to neighbouring droplets, position in the well and droplet size

314 (past a threshold) do not affect the droplet growth rate (Supplementary figures 14-17).

315 We varied reaction and diffusion conditions as shown in Figure 6A. Active droplets formed

316 from $2 \mathrm{mM}$ substrate (ADP) grow 20x faster than passive droplets (1.24 versus $0.06 \mu \mathrm{m} \mathrm{h}^{-1}$, see

317 Supplementary table 1); droplets can grow 100x faster than passive droplets when ADP is increased

318 to $3 \mathrm{mM}$. Higher $\mathrm{K}_{72}$ concentration indeed accelerate growth, but at $40 \mu \mathrm{M}$ there is a reversal in

319 the effect, which we attribute to a rising droplet count (Figure 6B). The increase in droplet count,

320 although also a feature of an active system, competes with growth. Similarly, when protein

321 concentration is low $\left(10 \mu \mathrm{M} \mathrm{K}_{72}\right)$, we observe maximal growth rate at the lowest enzyme

322 concentration tested. The increase in enzyme concentration from 0.10 to $0.42 \mu \mathrm{M}$ is also

323 accompanied by an increase in the initial number of droplets, that we cannot control in our setup.

324 The solution reaches supersaturation more rapidly, which facilitates widespread nucleation of

325 multiple nuclei that then grow limited by diffusion, rather than growth or localized nucleation

326 around some seeding droplets, and the measure growth rate is lower. ${ }^{33}$ When enzyme concentration

327 is varied and the protein concentration is higher $\left(20 \mu \mathrm{M} \mathrm{K}_{72}\right)$, the optimal enzyme concentration

328 for growth also shifts to a higher value $(0.42 \mu \mathrm{M})$. The complex balance between the two phases,

329 and the two processes (reaction and diffusion), may result in two distinct active droplet regimes -

330 nucleation-dominated or growth-dominated - but both are relevant as protocell models (Figure 6C).

331 The fact that we obtain significantly different growth rates by varying substrate, catalyst or

332 building block concentration means that our protocell model can have different fitness depending

333 on its composition and the environmental conditions. This is crucial for research aiming to achieve

334 Darwinian evolution with populations of artificial cells. ${ }^{34,35}$ We tested this feature by subjecting

335 two different populations to the same environmental conditions: one composed of $\mathrm{K}_{72}$, ADP and a 
seeding concentration of ATP, enough to have droplets from the start; and another mixture where

337 the seeding ATP was replaced by RNA oligomer ((ACGU)6), which also phase separates with $\mathrm{K}_{72}$

338 (Supplementary figure 18A). The enzyme PyK has a similar partitioning in the RNA-containing

339 droplets $\left(K_{p}=18\right.$, Supplementary figure 18B), but RNA displaces ADP in the droplets, ${ }^{36}$ so we

340 expected lower growth rates. Indeed, although the RNA-droplets start larger, they grow at 5x

341 smaller rates than the ATP-only droplets. RNA-containing droplets could be designed to grow faster

342 by using an enzyme with preference for RNA droplets, or by making use of RNA's catalytic

343 capacity. ${ }^{8,37}$

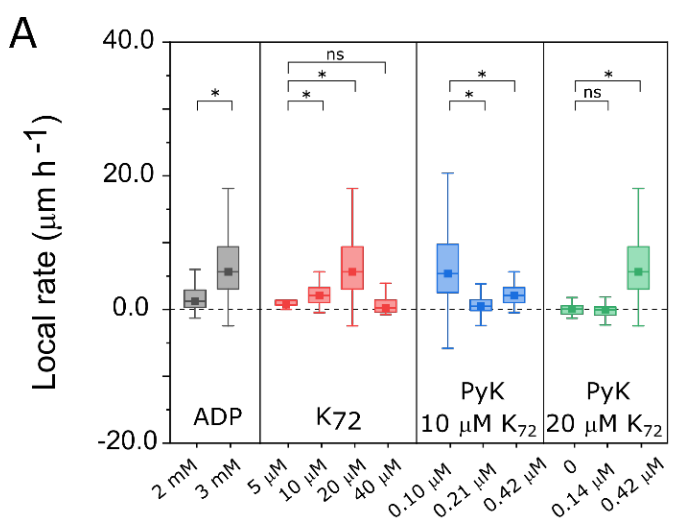

C

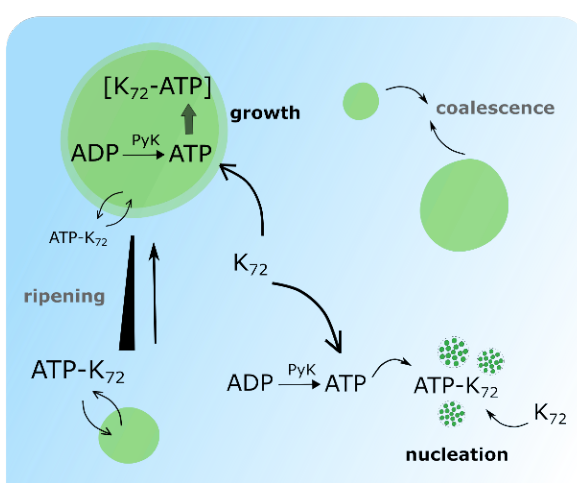

B

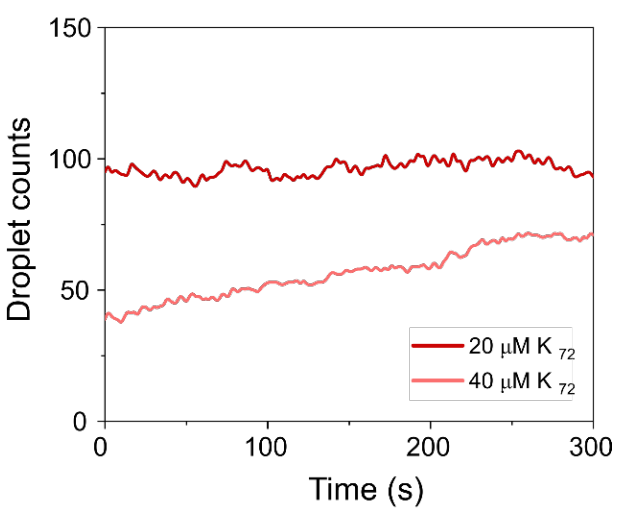

D

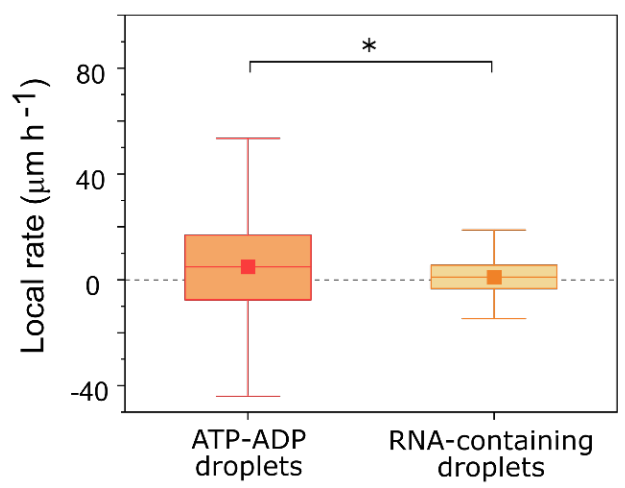

Figure 6. Growth rate of active droplets. (A) Growth rate dependence on different reaction-diffusion conditions. The local rate was measured for all droplets in a frame within $200 \mathrm{~s}$ of experiment. In different experiments, the concentration of ADP, $\mathrm{K}_{72}$ and PyK was varied. Conditions for the ADP series: $\mathrm{K}_{72} 20 \mu \mathrm{M}$, PyK 5 units $/ \mathrm{mL}$, PEP 3 $\mathrm{mM}$. $\mathrm{K}_{72}$ series: ADP $3 \mathrm{mM}$, PyK 5 units $/ \mathrm{mL}$, PEP $3 \mathrm{mM}$. PyK series: ADP $3 \mathrm{mM}$, PEP $3 \mathrm{mM}$ and $\mathrm{K}_{72}$ as informed on the plot. Conditions common to all sets shown: $\mathrm{MgCl}_{2} 0.5 \mathrm{mM}$ and HEPES $50 \mathrm{mM}, \mathrm{pH}$ 7.4, sample size $20 \mu \mathrm{L}$. The experiment for $3 \mathrm{mM}$ ADP, $20 \mu \mathrm{M} \mathrm{K}_{72}$ and 5 units/mL PyK appears in more than one series. (B) Droplet count during the growth phase of two of the experiments depicted in (A). (C) Active droplets grow around seeding droplets or also nucleate in a supersaturated solution of $\mathrm{K}_{72}$. (D) Active droplets of different compositions grow at significantly different rates. 


\section{Discussion}

355 We developed a protocell model that mimics two key features of cellular growth: the volume 356 expansion with a constant protocell count and the intrinsic relation between content and size. The 357 ATP-K $\mathrm{K}_{72}$ coacervates grow as result of a reaction that converts ADP into droplet-forming ATP, 358 catalyzed by pyruvate kinase. The catalyst is an important component, that due to its efficiency and 359 lack of side reactions, allows for a fine control of ATP formation. Although the use of an enzyme 360 may seem to decrease the prebiotic relevance of our model, we argue that the active coacervate 361 droplets do not rely on any specific interaction and the principles found here can be applied to any 362 complex coacervate.

Several works have pointed out the lack of a membrane as a disadvantage of coacervates as

364 protocellular models. ${ }^{38,39}$ Indeed membrane-less droplets are prone to (accelerated) Ostwald ripening and have no barrier to prevent fusion, but we found that complex coacervate droplets are remarkably stable. Unlike commonly studied emulsions, complex coacervate droplets are held 367 together by attractive electrostatic interactions. We show that the magnitude of the electric 368 attraction between a droplet and a departing soluble component like $\mathrm{K}_{72}$ may compensate the 369 driving force of Laplace pressure from small to larger droplets. Therefore, complex coacervate 370 emulsions can remain stable for hours without showing any sign of Ostwald ripening. More than a 371 technical advantage that allows us to measure growth rates without the competition of ripening, 372 this is a requirement for a growing protocell - "before replicators and reproducers, there must be 373 survivors". 40 An advantage of our approach is that we are able to follow individual droplets. This allows to separate the contribution of (rare) fusion events from steady, active growth; and additionally, to 376 obtain a precise profile of droplet sizes and to evaluate the influence of reaction rates and 377 environmental factors on the growth rate of droplets. Most active droplet studies so far have 378 focused on droplet count and average size, which are more susceptible to the interference of droplet 379 motion. ${ }^{15,41}$ Based on individual droplet traces, we found that our fuel-driven active droplet grow 
380 by diffusion, in a classical nucleation-growth fashion, ${ }^{33}$ but that the rate is determined by the ATP-

381 forming reaction. As a result, droplet radius has a $t^{1 / 2}$ dependency, and the speed can be controlled 382 by substrate, catalyst and protein concentrations. Moreover, the growth profile shows that liquid383 liquid phase separation alters the overall kinetics of the kinase reaction, by introducing a positive 384 feedback where larger droplets have an increased enzyme and ADP copy number, similar to the 385 effect of physical autocatalysis. ${ }^{42}$

386 Growth and survival are, ultimately, properties of a population, and we show that we can 387 use our model system to create populations with distinct growth rates, which can lead to distinct 388 fitness. From microscopy experiments where the droplets do not need to be immobilized or 389 stabilized, we extract growth rates of all droplets in both populations and found that RNA390 containing droplets grow $5 \mathrm{x}$ more slowly than the original ATP-K 72 droplets, which can be 391 rationalized in terms of the partitioning of ADP and therefore, the strength of the positive feedback 392 in the kinase reaction. We point out that the eventual slowing down of growth is not an intrinsic 393 property of active coacervates, but a consequence of the limited amount of $\mathrm{K}_{72}$ and PyK. We 394 envision that by designing systems with a higher catalytic efficiency in the presence of RNA, and 395 by introducing a common substrate supply, this is a first step towards competition and evolution of 396 active coacervate protocells. 
398 Materials and solution compositions. For the coacervates preparation, magnesium chloride anhydrous, sodium 399 chloride, ATP disodium salt, ADP disodium salt and pyruvate kinase type VII from rabbit muscle (EC 2.7.1.40, 2.8

$400 \mathrm{mg} \mathrm{mL} \mathrm{m}^{-1}$, ca. 1400 units $\mathrm{mL}^{-1}$, molecular weight used: $223 \mathrm{kDa}$ - tetramer) were purchased from Sigma-Aldrich; HEPES 401 free acid and phosphoenolpyruvate monopotassium salt were purchased from FluoroChem. For the microscopy 402 chambers: methoxy PEG silane (MW 5000) was purchased from JenKem Technology USA and 8 or 18 wells 403 chambered $\mu$-slides with glass bottom (No. 1.5 polymer coverslip) were acquired from Ibidi. For enzyme labeling, 404 Alexa Fluor $647 \mathrm{C}_{2}$ maleimide was purchased from Fischer Scientific. For HPLC experiments, potassium phosphate 405 mono and dibasic salts were purchased from Sigma-Aldrich.

The following stock solutions were prepared by dissolving or diluting in MilliQ: $500 \mathrm{mM}$ and $100 \mathrm{mM}$ HEPES pH 7.4 (adjusted with NaOH $6 \mathrm{M}$ ), $10 \mathrm{mM} \mathrm{MgCl}_{2}, 1 \mathrm{M} \mathrm{NaCl}, 100$ mM ADP, $100 \mathrm{mM}$ ATP, pyruvate kinase $1 \mathrm{mg} \mathrm{mL}{ }^{-1}$. A $100 \mathrm{mM}$ PEP solution was prepared in the $500 \mathrm{mM}$ HEPES. All of the latter were stored at $-20^{\circ} \mathrm{C}$ for no longer than a month. mPEG silane was dissolved and sonicated in dry DMSO to a $30 \mathrm{mg} \mathrm{mL}^{-1}$ concentration, and the stock kept for no longer than a week at room temperature. Alexa Fluor 647 NHS ester was dissolved in dry DMF

411 to a concentration of $10 \mathrm{mg} \mathrm{mL}^{-1}$ and kept at $-20^{\circ} \mathrm{C}$.

413 Pyruvate kinase labeling. We followed Thermo-Fischer instructions: $100 \mu \mathrm{L}$ of enzyme stock, directly as purchased 414 (PyK $2.8 \mathrm{mg} \mathrm{mL}^{-1}$ or ca. $12 \mu \mathrm{M}$ ), were mixed with $100 \mu \mathrm{L}$ of HEPES $0.1 \mathrm{M}$ to reach pH 7 and a concentration of ca. $4156 \mu \mathrm{M}$. Disulfide bonds were reduced by adding a large excess of DTT ( $2 \mu \mathrm{L}$ of a $0.1 \mathrm{M}$ stock); the excess was removed 416 after 30 minutes by centrifugal filtering (MWCO $3 \mathrm{kDa}, 2 \mathrm{~mL}$, Centricon, Merck) with degassed HEPES buffer, until 417 the volume reached ca. $200 \mu \mathrm{L}$ again. Alexa Fluor- $647 \mathrm{C}_{2}$ maleimide was freshly dissolved in $\mathrm{DMF}\left(10 \mathrm{mg} \mathrm{mL}^{-1}\right.$ or $4187.7 \mathrm{mM}$ stock) and $1.5 \mu \mathrm{L}$ were added to the mixture (final $60 \mu \mathrm{M}$ of dye, or 10 equiv. in regards to PyK tetramer). 419 The mixture was placed on a thermoshaker for 2 hours, at $600 \mathrm{rpm}$ and room temperature (ca. $21^{\circ} \mathrm{C}$ ). For removal of 420 unreacted dye, the reaction mixture was diluted to $2 \mathrm{~mL}$ with phosphate buffer (20 $\mathrm{mM}, \mathrm{pH} 7)$ and transferred to a 421 previously wetted centrifugal filter (MWCO $3 \mathrm{kDa}, 2 \mathrm{~mL}$, Centricon, Merck). Following fabricator instructions, the mixture was centrifuged at $500 \times \mathrm{g}$ for 30 minutes at $4{ }^{\circ} \mathrm{C}$. Until the filtrate was colorless and $50 \mu \mathrm{L}$ in volume, the 423 following steps were repeated: re-suspend with a pipette, dilute to $2 \mathrm{~mL}$ with phosphate buffer, and centrifuge. The 424 flow-through was kept for control experiments, and the enzyme solution was further purified by dialysis against 14 $\mathrm{mL}$ of MilliQ overnight (Thermo Scientific ${ }^{\mathrm{TM}}$ Slide-A-LyzerTM MINI Dialysis Device, 3.5K MWCO, 2 mL). 
Phase diagram. Coacervation of $\mathrm{K}_{72}$ and nucleotides ADP or ATP was always assessed with a commonly used turbidity assay, combined with microscopy. The absorbance at $600 \mathrm{~nm}$ was measured using a plate reader Spark M10 (Tecan), for samples containing: $25 \mathrm{mM}$ HEPES pH 7.4, $20 \mu \mathrm{M} \mathrm{K}_{72}, 1 \mathrm{mM} \mathrm{MgCl}_{2}$ and a varying concentration of ADP or ATP ranging from 1-10 mM. The samples were prepared in a $30 \mu \mathrm{L}$ scale and placed in a 384-well plate (Nunc, flat bottom). Absorbance (Abs) was measured before and after $2 \mu \mathrm{L}$ additions of $\mathrm{NaCl} 0.5 \mathrm{M}$, until it reached the value of

431 the control lacking any nucleotide. Turbidity $(\%)$ was calculated as $100\left(1-10^{-\mathrm{Abs}}\right)$. Critical salt concentration was calculated using the last three values of absorbance measured to extrapolate the concentration needed for Abs $=0$

433 (relative to the control).

435 Partitioning coefficients. Partitioning of $\mathrm{K}_{72}$, which always contains the GFP label, and of pyruvate kinase was calculated via confocal microscopy. The active coacervates were prepared in the default composition, and $1 \%$ volume 437 of Alexa 647-labeled pyruvate kinase (as obtained after purification) was added to the mixture. The averaged intensity of GFP and Alexa 647 emission was calculated for multiple droplets. A blank for both channels was obtained with a 439 sample containing only buffer, and the averaged intensity taken as background intensity. The partitioning coefficient 440 of the protein or the enzyme was then calculated as $K_{p}=\left(I_{\text {coacervate }}-I_{\text {background }}\right) /\left(I_{\text {dilute phase }}-I_{\text {background }}\right) . K_{p}$ of labeled 441 pyruvate kinase was considered to represent the $\mathrm{K}_{\mathrm{p}}$ of un-labeled enzyme.

443 coacervates in their default composition were prepared, but now PEP and ADP were added as well (3 mM each), in a 444 total volume of $100 \mu \mathrm{L}$. The sample was centrifuged for $30 \mathrm{~min}$, after which the coacervate phase (cc) can be seen as 445 a pellet at the bottom of the Eppendorf. The dilute phase (dp) was removed, avoiding as much as possible to collect coacervate phase (cp) as well. The pellet was dissolved with $30 \mu \mathrm{L}$ of $\mathrm{NaCl} 1 \mathrm{M}$, and then pipetted back to measure its 447 volume. Both phases were then analysed using a Shim-pack WAX-1 column (particle size $5 \mu \mathrm{M}, 4.6 \times 50 \mathrm{~mm}$, 448 Shimadzu), at $1 \mathrm{~mL} \mathrm{~min}^{-1}$ flow and $45^{\circ} \mathrm{C}$, using a gradient $0-100 \%$ B in 15 minutes (A: potassium phosphate buffer $449 \mathrm{pH}$ 7, $20 \mathrm{mM}$; B: potassium phosphate buffer $\mathrm{pH}$ 7, $480 \mathrm{mM}$ ). The peaks in the $254 \mathrm{~nm}$-chromatogram with retention 450 times of 10.0 and 12.4 min were identified as ADP and ATP, respectively. The peak in the $215 \mathrm{~nm}$-chromatogram with 451 retention time 9.5 min corresponds to PEP. The partitioning coefficient was then calculated as $K_{p}=$ peak_area $_{c p} \mathrm{x}$ dilution_factor $\mathrm{cp}_{\mathrm{cp}} / \mathrm{peak}_{-} \operatorname{area}_{\mathrm{dp}} \mathrm{x}$ dilution_factor $\mathrm{dp}$.

454 Microscopy chambers preparation. The Ibidi $\mu$-slides were functionalized with methoxy-PEG to minimize splashing 455 of the coacervate droplets and allow a more accurate measurement of radius over time. The protocol was adapted from Gidi, ACS App Mat 2018. Methoxy-PEG silane (MW 5000) was added to dry DMSO (30 mg mL $\mathrm{mL}^{-1}$, ca. $20 \mu \mathrm{L}$ per well 
to be functionalized) and placed in a thermoshaker at $60^{\circ} \mathrm{C}$. While it dissolved completely, the $\mu$-slides were cleaned

458 thoroughly: washed with dilute detergent, distilled water and ethanol, and dried with pressurized air; then placed in a

459 plasma cleaner (in a usual cleaning cycle according to fabricator instructions) or an ozone cleaner. This removes

460 adsorbed particles, making all hydroxyl groups available for bonding with the PEG silane. The slide was then placed

461 in the oven at $60^{\circ} \mathrm{C}$ to prevent precipitation when the PEG silane solution comes into contact with the glass. Finally,

462 the solution was added to each well, the slide was placed in a covered glass Petri dish, and the Petri dish inside an oven

463 at $60{ }^{\circ} \mathrm{C}$. After 2 hours, the slide was washed thoroughly with ethanol, MilliQ water (with sonication for $5 \mathrm{~min}$ ) and

464 ethanol, then dried with pressurized air and placed in an oven to dry completely. The slides were used the day after, for a maximum of 2 weeks or surface defects start to be observed.

Image and video acquisition. Images and time lapses were recorded at room temperature on a CSU X-1 Yokogawa spinning disc confocal unit connected to an Olympus IX81 inverted microscope, using a 100x piezo-driven oil immersion objective (NA 1.3) and a $488 \mathrm{~nm}$ laser beam. Emission was measured at 500-550 nm, with $100 \mathrm{~ms}$ of exposure time, at a rate of 30 frames per minute, using an Andor iXon3 EM-CCD camera. The acquired images have a pixel size of $141 \mathrm{~nm}$.

Indicated samples were recorded on a Liachroic SP8 confocal inverted microscope (Leica Microsystems,

$474(0.75 \mathrm{NA})$ or a $10 \mathrm{x}$ air $(0.45 \mathrm{NA})$ objective, depending on the nature of the droplets. For the GFP channel, $0.6 \%$ of the nominal power of a cyan laser @ $488 \mathrm{~nm}$ and a normal PMT detector were used, measuring at 493-620 nm, with a gain of $600 \mathrm{~V}$ and an offset of $-0.1 \%$. For the Alexa- 647 channel, 1.5\% of the total power of a red laser @ $638 \mathrm{~nm}$ and HyD SP GaAsP detector in Standard mode acquiring at $658-779 \mathrm{~nm}$ were used. Images were acquired at a rate of 12-30 frames per minute and have a pixel size of $377 \mathrm{~nm}$ or $1.88 \mu \mathrm{m}$ depending on the objective.

Active coacervates experiments. All samples were prepared just before an experiment, usually in a $20 \mu \mathrm{L}$ size; the components were kept in ice during preparation, but not the mixture. Active coacervates had the default composition 482 of (in order of addition): $50 \mathrm{mM}$ HEPES pH 7.4, $0.5 \mathrm{mM} \mathrm{MgCl}_{2}, 3 \mathrm{mM}$ ADP, $20 \mu \mathrm{M} \mathrm{K} \mathrm{K}_{72}, 0.42 \mu \mathrm{M}$ pyruvate kinase and $3 \mathrm{mM}$ PEP. For investigating the effect of kinase activity, substrate concentration and protein diffusion on growth rate, the default concentrations were used, but the following were changed, respectively: the enzyme concentration was varied ranging from $0.1-0.42 \mu \mathrm{M}$, PEP was varied from 1-3 mM, or $\mathrm{K}_{72}$ was varied from 5-40 $\mu \mathrm{M}$. A negative control without enzyme was performed. See Supplementary Table 1 for the full list of conditions. 
Passive coacervates and Ostwald ripening controls. Passive $\mathrm{K}_{72}$-ATP coacervates were used as negative controls

489 for growth, and contained (in order of addition): $50 \mathrm{mM}$ HEPES pH 7.4, 1-3 mM ATP, $20 \mu \mathrm{M} \mathrm{K}_{72}$ and $0.5 \mathrm{mM} \mathrm{MgCl}_{2}$

490 The mixtures were prepared directly in the passivated microscopy chamber, and covered with a glass slide before

491 recording 1-hour long videos. Oil droplets were used as positive controls for Ostwald ripening, and prepared at $2 \% \mathrm{v} / \mathrm{v}$

492 fractions, in the presence of 2\% v/v SDS and Nile Red as fluorescent dye. We chose 1-bromo-dodecane and 1-bromo-

493 propane based on their densities and solubilities.

495 Competition assay. The two droplet populations were analyzed separately, but prepared with the same enzyme and protein stocks. The slow growing population was based on our default system: $50 \mathrm{mM}$ HEPES pH 7.4, $3 \mathrm{mM}$ ADP, $49720 \mu \mathrm{M} \mathrm{K}_{72}$ and $0.5 \mathrm{mM} \mathrm{MgCl}$, with the difference of $1 \mathrm{mM}$ ATP being added to pre-nucleate droplets. The fast growing population was composed of: $50 \mathrm{mM}$ HEPES pH 7.4, $3 \mathrm{mM} \mathrm{ADP,} 10 \mu \mathrm{M} \mathrm{Cy}_{5}-(\mathrm{ACGU})_{6} \mathrm{RNA}$ oligomer, 20 $\mu \mathrm{M} \mathrm{K}_{72}$ and $0.5 \mathrm{mM} \mathrm{MgCl}_{2}$.

501 Pyruvate kinase activity. Enzyme activity in the presence of coacervates was determined by measuring ATP concentration in the emulsion as whole, at different reaction times. Ten copies of the active coacervates (default composition) were prepared, and for each copy the reaction was quenched at a different time, using acetic acid (to $\mathrm{pH}$ 2 , or $1 \% \mathrm{v} / \mathrm{v}$ ). Conveniently, the low $\mathrm{pH}$ also dissolves the coacervates. The analysis was done by HPLC, using the same column and run as described in Partitioning coefficients. The control experiment was a sample of equal composition, with the addition of $100 \mathrm{mM} \mathrm{NaCl}$ to dissolve existing $\mathrm{K}_{72}$-ADP coacervates, and prevent formation of $\mathrm{K}_{72}$-ATP coacervates.

Quantitative video analysis. Raw fluorescence confocal microscopy videos were processed and analyzed with

511 for background emission and prepare the video for edge detection; performs edge detection of objects on each frame

512 with a canny operator, with thresholds customized per video; labels the objects based on their centroid and extracts 513 area, circularity and pixel intensity. Across frames, the script compares centroids to distinguish between fusion, settling 514 and growing events. We select relevant droplets based on an aspect ratio $<2.5$ and on a minimum number of 30 frames 515 accurately tracked. The properties are then analyzed in a second pipeline that lists properties such as area, radius, volume and pixel intensity, per droplet, and per frame. It also determines the slope of the radius versus time curve in 517 intervals of 10 frames, after outliers are removed with a moving average interpolation. 
Statistical analysis. The boxes contain the 25 and $75 \%$ percentiles and the median as the square dot on the median

520 line (actual values in Error! Reference source not found.). The whiskers represent the minimal and maximal values

521 measured and outliers are omitted. The difference between the results is significantly different if $\mathrm{p}<0.05$ in a Mood's

522 median test.

$524 \mathbf{K}_{72}$ expression and purification. We adapted the procedure previously described by Pesce et al and Te Brinke et $a l{ }^{24,25}$ BL21(DE3) cells were transformed with the pET25-sfill-K $\mathrm{K}_{72}$ plasmid. Expression was performed in Terrific Broth medium (TB; $12 \mathrm{~g} \mathrm{~L}^{-1}$ tryptone and $24 \mathrm{~g} \mathrm{~L}^{-1}$ yeast autolysate) enriched with phosphate buffer $\left(2.31 \mathrm{~g} \mathrm{~L}^{-1}\right.$ potassium phosphate monobasic and $12.54 \mathrm{~g} \mathrm{~L}^{-1}$ potassium phosphate dibasic), glycerol (4 mL per $\left.1 \mathrm{~L} \mathrm{~TB}\right)$, glucose $(0.1 \mathrm{wt} \%)$ and $100 \mu \mathrm{g} \mathrm{mL}^{-1}$ ampicillin. Because of the glycine- and lysine-rich nature of $\mathrm{K}_{72}$, the $\mathrm{TB}$ was supplemented with $0.10 \mathrm{~g}$ of amino acids per $1 \mathrm{~L}$ of TB. The bacterial cultures were grown at $37{ }^{\circ} \mathrm{C}$ till an optical density $\mathrm{OD}_{600}$ reached saturation $(1.5-1.8)$, subsequently cells were cooled to $18^{\circ} \mathrm{C}$ to allow expression overnight. Cells were pelleted at 5,000 $\mathrm{g}$ and resuspended in lysis buffer consisting of $10 \mathrm{mM}$ Tris, $300 \mathrm{mM} \mathrm{NaCl}, 20 \mathrm{mM}$ imidazole, $\mathrm{pH} 8$, supplemented with 1x complete protease inhibitor cocktail (Roche). Cells were disrupted through sonication on ice and cleared by centrifugation at $20,000 \mathrm{~g}$ at $4{ }^{\circ} \mathrm{C}$.

His-tag labelled $\mathrm{K}_{72}$ was purified from the soluble fraction with a HisTrap column (GE Healthcare, elution

$536 \mathrm{mM}$ Tris $\mathrm{pH} 8,300 \mathrm{mM} \mathrm{NaCl}$ ), the protein was concentrated to $2-4 \mathrm{~mL}$ using a Vivaspin 15 concentrator (MWCO of

$53730 \mathrm{kDa}$ ). Then the protein was passed through a S200 SEC column (GE-Healthcare). Protein purity was analyzed by

538 SDS-PAGE using a 4-20\% mini-Protean gel (Bio-Rad) stained with instant blue, pure $\mathrm{K}_{72}$ fractions with corresponding size were combined and dialyzed against MilliQ. $\mathrm{K}_{72}$ stock solution was obtained by concentrating the protein using a

540 Vivaspin 15 concentrator (MWCO $30 \mathrm{kDa}$ ) till the protein reached a concentration of $80 \mu \mathrm{M}$. Aliquots of the stock 541 solution were snap frozen and stored at $-80^{\circ} \mathrm{C}$.

\section{Acknowledgments}

544 We thank Dr. Ioannis Alexopoulos (Radboud University) for helpful advice on recording the droplets in a confocal microscope, and Anne-Déborah Nguindjel, Dr. Jessie van Buggenum and Dr. the additional experiment with PDDA-PSPMA coacervates. The authors acknowledge financial 
550 E.S. designed and supervised the project. K.K.N. designed, performed and analyzed kinetics and microscopy experiments. M.H.I.vH performed active droplets experiments and FRAP. A.A.M.A expressed and purified the $\mathrm{K}_{72}$ protein. I.R. and E.S. wrote the MatLab script for analysis. K.K.N. and E.S. wrote the manuscript, with input and revisions from all authors.

\section{Additional information}

Supplementary Information accompanies this paper.

\section{Competing Interests}

The authors declare no competing interests.

\section{References}

562 1. Szathmáry, E. \& Smith, J. M. The major evolutionary transitions. Nature 374, 227-232 (1995).

563 2. Chen, I. A. GE prize-winning essay: The emergence of cells during the origin of life. Science 314, 1558-1559 $564 \quad$ (2006).

565 3. Qiao, Y., Li, M., Booth, R. \& Mann, S. Predatory behaviour in synthetic protocell communities. Nat. Chem. provides a model for protocells. Nat. Phys. 13, 408-413 (2017).

5. Wurtz, J. D. \& Lee, C. F. Chemical-Reaction-Controlled Phase Separated Drops: Formation, Size Selection, and Coarsening. Phys. Rev. Lett. 120, 1-25 (2018).

6. Weber, C. A., Zwicker, D., Jülicher, F. \& Lee, C. F. Physics of active emulsions. Reports Prog. Phys. 82, (2019).

7. Beneyton, T. et al. Out-of-equilibrium microcompartments for the bottom-up integration of metabolic functions. Nat. Commun. 9, 1-10 (2018).

8. Drobot, B. et al. Compartmentalised RNA catalysis in membrane-free coacervate protocells. Nat. Commun. 9, (2018).

9. Tena-Solsona, M. et al. Accelerated Ripening in Chemically Fueled Emulsions. ChemSystemsChem 2, 1-11 (2020).

10. Astoricchio, E., Alfano, C., Rajendran, L., Temussi, P. A. \& Pastore, A. The Wide World of Coacervates: From the Sea to Neurodegeneration. Trends Biochem. Sci. 45, (2020).

11. Ghosh, B., Bose, R. \& Tang, T.-Y. D. Can coacervation unify disparate hypotheses in the origin of cellular life. Curr. Opin. Colloid Interface Sci. (2020).

12. Nakashima, K. K., Vibhute, M. A. \& Spruijt, E. Biomolecular chemistry in liquid phase separated compartments. Front. Mol. Biosci. 6, (2019).

13. Zhu, T. F. \& Szostak, J. W. Coupled growth and division of model protocell membranes. J. Am. Chem. Soc. 131, 5705-5713 (2009).

14. Ivanov, I. et al. Directed Growth of Biomimetic Microcompartments. Adv. Biosyst. 3, 1-9 (2019).

15. Deshpande, S. et al. Spatiotemporal control of coacervate formation within liposomes. Nat. Commun. 10, 111 (2019).

16. Takakura, K., Toyota, T. \& Sugawara, T. A novel system of self-reproducing giant vesicles. J. Am. Chem. Soc. 
125, 8134-8140 (2003).

17. Berry, J., Weber, S. C., Vaidya, N., Haataja, M. \& Brangwynne, C. P. RNA transcription modulates phase transition-driven nuclear body assembly. Proc. Natl. Acad. Sci. U. S. A. 112, E5237-45 (2015).

18. Campbell, A. Synchronization of cell division. Bacteriol. Rev. 21, 263-272 (1957).

19. Nakashima, K. K., Baaij, J. F. \& Spruijt, E. Reversible generation of coacervate droplets in an enzymatic network. Soft Matter 14, 361-367 (2018).

20. Bracha, D. et al. Mapping Local and Global Liquid Phase Behavior in Living Cells Using PhotoOligomerizable Seeds. Cell 175, 1467-1480.e13 (2018).

21. Koga, S., Williams, D. S., Perriman, A. W. \& Mann, S. Peptide-nucleotide microdroplets as a step towards a membrane-free protocell model. Nat. Chem. 3, 720-4 (2011).

22. Frankel, E. A., Bevilacqua, P. C. \& Keating, C. D. Polyamine/Nucleotide Coacervates Provide Strong Compartmentalization of Mg2+, Nucleotides, and RNA. Langmuir 32, 2041-2049 (2016).

23. Aumiller Jr, W. M. \& Keating, C. D. Phosphorylation-mediated RNA/peptide complex coacervation as a model for intracellular liquid organelles. Nat. Chem. 8, 129-137 (2015).

24. Brinke, E. et al. Dissipative adaptation in driven self-assembly leading to self-dividing fibrils. Nat. Nanotechnol. 13, 849-856 (2018).

25. Pesce, D., Wu, Y., Kolbe, A., Weil, T. \& Herrmann, A. Enhancing cellular uptake of GFP via unfolded supercharged protein tags. Biomaterials 34, 4360-4367 (2013).

26. Yang, H. et al. Tuning Ice Nucleation with Supercharged Polypeptides. Adv. Mater. 28, 5008-5012 (2016).

27. Zwicker, D., Hyman, A. A. \& Julicher, F. Suppression of Ostwald ripening in active emulsions. Phys. Rev. E - Stat. Nonlinear, Soft Matter Phys. 92, 1-13 (2015).

28. Dine, E., Gil, A. A., Uribe, G., Brangwynne, C. P. \& Toettcher, J. E. Protein Phase Separation Provides LongTerm Memory of Transient Spatial Stimuli. Cell Syst. 6, 655-663 (2018).

29. Erickson, H. P. Size and shape of protein molecules at the nanometer level determined by sedimentation, gel filtration, and electron microscopy. Biol. Proced. Online 11, (2009).

30. Nakashima, K. K., André, A. A. M. \& Spruijt, E. Enzymatic control over coacervation. Methods Enzymol. 646, 353-389 (2020).

31. Oria-Hernández, J., Cabrera, N., Pérez-Montfort, R. \& Ramírez-Silva, L. Pyruvate kinase revisited: The activating effect of K+. J. Biol. Chem. 280, 37924-37929 (2005).

32. Adamski, P. et al. From self-replication to replicator systems en route to de novo life. Nat. Rev. Chem. 4, (2020).

33. Shin, Y. et al. Liquid Nuclear Condensates Mechanically Sense and Restructure the Genome. Cell 175, 14811491.e13 (2018).

34. Adamala, K. \& Szostak, J. W. Competition between model protocells driven by an encapsulated catalyst. Nat. Chem. 5, 495-501 (2013).

35. Chen, I. A., Roberts, R. W. \& Szostak, J. W. The emergence of competition between model protocells. Science 305, 1474-1476 (2004).

36. Cakmak, F. P., Choi, S., Meyer, M. C. O., Bevilacqua, P. C. \& Keating, C. D. Prebiotically-relevant low polyion multivalency can improve functionality of membraneless compartments. Nat. Commun. 11, 1-11 (2020).

37. Poudyal, R. R. et al. Template-directed RNA polymerization and enhanced ribozyme catalysis inside membraneless compartments formed by coacervates. Nat. Commun. 10, 1-13 (2019).

38. Mason, A. F., Buddingh, B. C., Williams, D. S. \& Van Hest, J. C. M. Hierarchical Self-Assembly of a Copolymer-Stabilized Coacervate Protocell. J. Am. Chem. Soc. 139, 17309-17312 (2017).

39. Deng, N. N. Complex coacervates as artificial membraneless organelles and protocells. Biomicrofluidics 14, (2020).

40. Szathmáry, E. \& Smith, J. M. From replicators to reproducers: The first major transitions leading to life. $J$. Theor. Biol. 187, 555-571 (1997).

41. Donau, C. et al. Active coacervate droplets as a model for membraneless organelles and protocells. Nat. Commun. 11, 1-10 (2020).

42. Bissette, A. J., Odell, B. \& Fletcher, S. P. Physical autocatalysis driven by a bond-forming thiol-ene reaction. Nat. Commun. 5, 1-8 (2014). 